Meta

Journal des traducteurs

Translators' Journal

\title{
Les signes sociaux et leur traduction
}

\section{Zhang Xinmu}

Volume 44, numéro 1, mars 1999

Théorie et pratique de la traduction en Chine

The Theory and Practice of Translation in China

URI : https://id.erudit.org/iderudit/004642ar

DOI : https://doi.org/10.7202/004642ar

Aller au sommaire du numéro

Éditeur(s)

Les Presses de l'Université de Montréal

ISSN

0026-0452 (imprimé)

1492-1421 (numérique)

Découvrir la revue

\section{Citer cet article}

Xinmu, Z. (1999). Les signes sociaux et leur traduction. Meta, 44(1), 110-120. https://doi.org/10.7202/004642ar

\section{Résumé de l'article}

Cet article propose une typologie des signes sociaux pour en montrer les caractéristiquessoumises à différents types de traitement : transfert des sens connotatifs des signessociolinguistiques, conservation de la saveur exotique des idiotismes et interprétation des signes coutumiers. d'utilisation que vous pouvez consulter en ligne.

https://apropos.erudit.org/fr/usagers/politique-dutilisation/ 


\title{
Les signes sociaux et leur traduction
}

\author{
zhang xinmu \\ Université de Nankin, \\ Nankin, Chine
}

\begin{abstract}
RÉSUMÉ
Cet article propose une typologie des signes sociaux pour en montrer les caractéristiques soumises à différents types de traitement: transfert des sens connotatifs des signes sociolinguistiques, conservation de la saveur exotique des idiotismes et interprétation des signes coutumiers.
\end{abstract}

\begin{abstract}
This paper proposes a typology of social signs to show their characteristics to which some types of treatments are advanced: to transfer connotated meanings of sociolinguistic signs, to preserve the exotic savour of idiom signs and to interprete customs signs.
\end{abstract}

La traduction est une opération complexe dont la problématique se manifeste sous divers aspects. Grâce à la linguistique moderne, et surtout à la sociolinguistique, nous avons pu bénéficier de nouvelles acquisitions de la recherche: un grand nombre de problèmes surgis dans l'activité traduisante ont trouvé des solutions relativement satisfaisantes. Toutefois, certains aspects de la traduction semblent plus ou moins négligés jusqu'ici ou traités d'une manière peu scientifique. Nous entendons par là que les éléments socioculturels du texte n'ont pas encore pris une place convenable dans les préoccupations des traducteurs. En ce sens, une approche des signes sociaux dans le cadre de la traduction devient nécessaire. Nous essayons ici de classer certaines catégories de signes sociaux, de démontrer leurs caractéristiques et de proposer des modalités de traduction.

\section{LES SIGNES SOCIAUX}

Pierre Guiraud (1983) divise les signes en trois grandes catégories: signes logiques, signes esthétiques et signes sociaux. Les signes logiques et les signes esthétiques reflètent les relations entre l'homme et la nature, tandis que les signes sociaux révèlent les rapports entre les hommes:

La communication sociale, elle, a pour objet de signifier la relation entre les hommes et par conséquent entre l'émetteur et le récepteur. La société est un système de relations entre les individus qui a pour but la procréation, la défense, les échanges, la production, etc. À cettefin, la situation des individus au sein du groupe et des groupes au sein d'une collectivité doit être signifiée. (Guiraud 1983: 97)

Les signes sociaux englobent, selon Guiraud, les signes d'identité, les signes de politesse, les rites, les modes, les jeux, etc. Les signes d'identité comprennent les armes, les totems, les uniformes, les décorations, les coiffures, les noms, les enseignes, les 
marques de fabrique, etc. Ces signes servent à reconnaître l'identité des individus et des groupes. Les signes de politesse comprennent la prosodie, les formules de politesse, les injures, la kinésique, la proxémique, la nourriture, etc. Les signes d'identité permettent de marquer l'appartenance d'un individu à un groupe ou à une fonction, les signes de politesse marquent la nature des relations entre les individus. Les rites sont des moyens de communication des groupes. Les cultes religieux, les rites d'initiation, les sacres, les cérémonies, les rites funéraires instituent des relations entre le groupe et l'individu qu'il reçoit dans son sein. Les modes sont des manières d'être propres au groupe: se vêtir, se nourrir, se loger, etc. Les jeux sont des imitations de la réalité sociale, ils correspondent aux trois grands modes de l'expérience humaine: intellectuelle et scientifique, pratique et sociale, affective et esthétique. Ce classement des signes sociaux ne prétend pas être le plus rationnel ou le plus complet, mais il nous permet de voir l'ampleur et la complexité de cette investigation.

Les signes peuvent être plus ou moins socialisés et conventionnalisés. Cependant, d'une manière générale, les signes sociaux le sont assez peu. C'est le cas des onomastiques, des insignes, des totems, des costumes, des métiers, des clans, etc. Chaque culture a son propre système. Ensuite, la plupart des signes sociaux sont du type motivé, soit par métaphore, soit par métonymie, ils sont fortement connotés, exprimant la majesté, la force, le pouvoir, I'aspiration ou l'humilité, et ces valeurs relèvent de la symbolique enracinée dans l'inconscient collectif. Dans la tradition chinoise, le gâteau delune, par exemple, par sa forme ronde, s'associe aux sens de la réunion et de la perfection; les cacahuètes, par leur caractère de procréation féconde, s'associent à l'idée de fécondité, etc. (Zhang 1989 : 24). De plus, par leur nature iconique, les signes sociaux s'apparentent aux signes esthétiques. Dans la communication sociale, l'émetteur est souvent porteur du signe et, en même temps, il est le référent de ce signe. Quand un soldat porte son uniforme, il émet un signe d'identité qui a pour référent ce soldat même. Cette confusion du sujet et de l'objet accentue le caractère esthétique des signes sociaux. La balance de la justice, l'olivier de la paix, à force de se voir attribuer un nouveau référent, perdent leur sens originel et entrent dans une symbolique relevant du domaine des arts et des littératures. Cela nous amène à réfléchir sur les signes sociaux utilisés dans le texte littéraire. Quels sont les signes sociaux dans le texte littéraire? Comment faut-il les classer? Sont-ils différents des signes sociaux hors du texte littéraire? Autant de questions que nous devons aborder si nous voulons connaître les systèmes de signes sociaux et leur fonctionnement dans la littérature. Les conclusions que nous en dégagerons serviront à mieux cerner l'essence du texte littéraire et amélioreront la qualité de la traduction.

\section{Les signes sociaux et la littérature}

Il est de notoriété publique que la littérature est un art du langage et qu'elle crée des objets linguistiques signifiants. Dans un texte littéraire coexistent les trois catégories de signes que nous venons d'évoquer. L'exemple de la casquette est très typique: elle pourrait être un objet ordinaire dont la fonction est de protéger le porteur contre le froid; elle pourrait être une marque sociale dont la fonction est d'indiquer son appartenance à un groupe social ; elle pourrait aussi, dans le cas de la casquette de Charles Bovary, être une image littéraire dont la fonction est de signifier la «gaucherie» du porteur (Zhang 1997: 19). Dans le premier cas, la casquette est un signe 
logique, arbitraire et conventionnalisé, son référent est unique; dans le second cas, elle devient un signe social, conventionnalisé, mais déjà moins arbitraire - il est rare qu'on se serve d'un foulard pour indiquer l'identité d'une personne, sauf dans le cas de signe esthétique; dans le troisième cas, elle est tout à fait un signe esthétique, implicite et analogique, elle exige une interprétation et non un décodage. Statistiquement parlant, les signes logiques sont surtout distribués au niveau de la langue, les signes sociaux et esthétiques au niveau du discours. Dans le discours, les signes sociaux et esthétiques se situent soit au niveau de l'histoire, soit au niveau de la narration. Du point de vue de la structure, les signes logiques sont intégrés dans les signes sociaux qui sont, à leur tour, intégrés dans les signes esthétiques. La valeur d'un signe linguistique ne sera définie que quand il s'intègre dans un niveau plus élevéque celui auquel il appartient.

Les signes sociaux sont, dans la plupart des cas, des unités d'indices, de nature intégrative,

I'unité renvoie alors, non à un acte complémentaire et conséquent, mais à un concept plus ou moins diffus, nécessaire cependant au sens de l'histoire: indices caractériels concernant les personnages, informations relatives à leur identité, notations d'atmosphère, etc. ; la relation de l'unité et son corrélat n'est plus alors distributionnelle, mais intégrative. (Barthes 1977: 20)

Pour comprendre un indice, il faut passer à un niveau supérieur, c'est-à-dire au niveau des actions ou de la narration. Cela veut dire que dans le texte littéraire, les signes sociaux se définissent moins par rapport à d'autres signes sociaux que par les signes de niveau différent, par le «contexte», comme on dit dans le langage courant. La sanction de ces signes se trouve «plus haut», hors du syntagme explicite, elle est plutôt paradigmatique. Les signes sociaux sont d'ailleurs métaphoriques et correspondent à une fonctionnalité de l'être.

Un personnage du roman, à supposer qu'il soit introduit par l'attribution d'un nom propre, se construit progressivement par des notations figuratives consécutives et diffuses le long du texte, et ne déploie sa figure complète qu'à la fin du roman, grâce à la mémorisation opérée par le lecteur. (Greimas 1973: 174)

Les signes d'identité et de rapport sont distribués le long du texte et aux différents niveaux du texte, ils forment un réseau d'indices ou une série de métaphores pour esquisser une structure des personnages en rapport.

$\mathrm{Si}$ les signes sociaux contribuent à peindre les personnages, ils sont aussi capables de décrire les aspects sociaux. Lucien Goldmann (1955: 16), avec son structuralisme génétique, met l'accent sur les relations entre l'œuvre littéraire et l'environnement socio-économique dans lequel l'œuvre a été produite. «Une idée, une œuvre n'aura sa vraie signification que quand elle est intégrée dans une vie, dans une action ${ }^{1}$. » La fête des Comices dans M adame Bovary nous présente un tableau de la vie sociale en Normandie au xixe siècle. Les signes sociaux sont nombreux: les décorations, les inscriptions, les cérémonies, les habits, les comportements, les échanges commerciaux, le festin, etc. Le discours du Conseiller est rempli de signes sociaux reflétant la monarchie:

Qu'il me soit permis, dis-je, de rendre justice à l'administration supérieure, au gouvernement, au monarque, M essieurs, à notre souverain, à ce roi bien-aimé à qui aucune branche de la prospérité publique ou particulière n'est indifférente, et qui dirige à la fois 
d'une main si ferme et si sage le char de l'État parmi les périls incessants d'une mer orageuse, sachant d'ailleurs faire respecter la paix comme la guerre, l'industrie, le commerce, l'agriculture et les beaux-arts².

Les signes sociaux ici sont du double type, ils sont à la fois logiques et affectifs. Les signes sociaux logiques indiquent la place de l'individu et du groupe dans la hiérarchie et dans I'organisation politique, économique, institutionnelle; les signes sociaux affectifs expriment les émotions, les sentiments que l'individu ou le groupe éprouve à l'égard des autres individus ou des autres groupes. Dans le texte littéraire, les signes sociaux logiques n'auront de valeur que quand ils sont associés aux signes sociaux affectifs (esthétiques), c'est-à-dire que leurs référents ne seront identifiés que quand ils s'intègrent aux niveaux supérieurs et dans le texte même.

\section{Typologie des signes sociaux dans le texte littéraire}

Nous venons de voir les signes sociaux généraux et les rapports que certains d'entre eux entretiennent avec la littérature. Ces signes posent le problème de compréhension pour les lecteurs de cultures différentes, d'abord pour le traducteur, puis pour le lecteur proprement dit. Nous allons essayer de classer, d'une manière peut-être arbitraire, les signes sociaux utilisés dans le texte littéraire et d'en dégager les caractéristiques qui pourraient gêner la compréhension et rendre difficile la traduction.

\section{Signes sociolinguistiques}

La sociolinguistique actuelle attire notre attention sur le contexte social du vocabulaire d'un peuple. Tyler (1969) nous rappelle l'existence des variantes du mot à l'intérieur d'une culture: il existe, au sein du groupe même, des variations d'un individu à l'autre ou d'un contexte à l'autre. Hymes, de son côté, souligne la nécessité d'une étude systématique des rapports entre la langue et la vie socioculturelle. Insistant sur le caractère dynamique de la communication, Hymes (1974) pense que

l'ethnosémantique tend à voir l'intégration de la langue et de la culture sous forme d'un code intégré dont la description se fait par des méthodes qui ne tiennent pas compte du comportement; l'ethnographie de la parole se soucie entre autres choses du déroulement de l'action, au cours duquel la structure et le sens naissent des rapports entre les codes et les circonstances.

Les variantes linguistiques se manifestent principalement sur trois plans: phonologique, grammatical et lexical.

Les variantes phonologiques se manifestent en général dans la conversation, mais aussi à l'écrit, et l'effet sonore du langage revêt souvent un sens sociologique: le fait de mettre «le» dans «si l'on... » marque un style recherché de l'auteur. Les rapports entre les éléments phonologiques et le texte littéraire sont évidents. Un poème présente des différences phonologiques repérables par rapport à un texte scientifique, il en est de même des effets sonores d'un roman qui diffèrent de ceux d'un drame. II faut envisager les problèmes phonologiques pendant l'opération traduisante et, en particulier, dans la traduction des poèmes.

Les variantes grammaticales semblent marquer la classe sociale du sujet parlant: les Noirs américains ont l'habitude d'omettre le prédicat ou ne mettent pas le «s» 
aux noms pluriels; les Chinois moyens ne se soucient pas tellement du sujet d'une proposition: dans un contexte défini, «je», «il» paraissent souvent superflus. $\mathrm{Si}$ l'auteur fait ressortir les variantes grammaticales, c'est qu'il veut mettre en relief les différences sociales. Cela constitue un aspect que nous devons cerner pendant la traduction.

Les variantes lexicales nous montrent des éléments sociaux et d'une manière plus sensible. Raymond Queneau (1982) nous montre comment un événement ordinaire peut être raconté de mille et une manières, avec un vocabulaire différent, avec une prosodie contrastée. Xu Jun (1992: 74) nous fait observer l'opposition des «tu» et «vous» utilisés par Stendhal. En fait, l'auteur laisse deviner une fissure dans l'amour entre Julien et $\mathrm{M}$ me de Rênal par l'emploi de «tu» ou «vous»; cet emploi n'est donc pas une simple désignation de sujet, mais connote le degré d'affection entre les personnages. Un signe linguistique engendre donc, dans son propre système, des signifiés différents selon la personne, le temps, le lieu et le contexte.

Dans le cas de deux langues, le cas où le traducteur convertit les signes d'une langue dans l'autre, le problème est encore plus complexe. Vu la différence dans la vie sociale, les signes linguistiques des deux langues, souvent, ne couvrent pas le même champ sémantique, les éléments lexicaux ne trouvent pas les mêmes référents d'une langue à l'autre. Au niveau des mots, nous avons pu remarquer la différence de découpage du champ sémantique. Le mot «paille» englobeen français la paille du blé et plus rarement la paille du riz; en chinois, les deux «pailles» sont désignés par deux mots ( 麦秸。稻草) tout à fait différents. Le temps joue aussi un rôle dans le découpage du champ sémantique: le mot «gendarme» en français désigne une personne portant l'arme et veillant à l'ordre public, le mot équivalent en chinois est «宪兵》 (soldat de la Constitution). Nous savons que la Constitution est une réalité récente en Chine et que le mot «gendarme» est apparu en français au xve siècle, il n'est donc pas convenable de prendre «gendarme» et «宪兵》 pour des équivalents. En outre, la même réalité peut être désignée d'une manière très différente selon le cadre social et naturel. Les Esquimaux semblent avoir plus de vingt mots pour désigner la couleur blanche, les Chinois d'avant Jésus-Christ avaient une vingtaine de caractères pour désigner la tortue ou le cheval, les Français continuent à allonger la liste des noms de fromages et de vins.

\section{Signes idiomatiques}

Les signes idiomatiques pourraient participer de cette catégorie. Un idiome est un ensemble de mots avec un désigné global, il ne peut trouver son équivalent dans une autre langue que globalement. Dans les œuvres littéraires, on voit souvent les expressions figées, les dictons, les locutions, les proverbes et les images archétypes qui révèlent les caractères socioculturels d'une langue. Ces éléments permettent non seulement de passer un message, mais aussi d'apporter des éléments socioculturels de la langue de départ. Les idiomes ne semblent pas être des signes de la communication directe dont le sens littéral est explicite et socialisé, ils sont plutôt des signes de signification dont le sens est implicite et médiatisé:

La distinction [...] entre la véritable communication et la simple manifestation, ou entre la communication et la signification, peut égal ement nous fournir la clé de la différence. 
[...] On interprète un indice, et l'interprétation en sera variable avec les récepteurs, selon leur intuition, leur compétence, etc. On décode un signal, et le décodage en est univoque pour tous les récepteurs en possession du code de communication. (Mounin 1970: 12-14)

Ces signes de signification, qui fournissent des «indices» socioculturels, constituent paradoxalement des obstacles de communication, les degrés de compréhension définissent les degrés d'interprétation. Ensuite, les idiomes sont porteurs de différences entre les langues. La langue n'est pas un élément passif dans la société, elle présente une pluralité d'intersections, compromis dynamique entre les groupes de pression biologiques, psychologiques et sociaux, d'où la nécessité de réaliser des ensembles de mots utilisés par une communauté linguistique donnée et ayant une existence historique et culturelle, c'est-à-dire un «idiome». En dernier lieu, nous remarquons que les idiomes sont perçus en unités inséparables. Si l'on dit en anglais «It's raining cats and dogs», cats and dogs est évidemment un ensemble inséparable, on ne peut trouver son équivalent que globalement dans une autre langue. II en est de même pour les idiomes suivants en français: le sel de la terre, la voie lactée, la brebis galeuse, pomme de discorde, etc.

Les mythes et mythologies constituent aussi une catégorie de signes socioculturels dans la littérature:

L'étude des mythes et thèmes chez les écrivains constitue un secteur del'histoire et de la critique littéraire qui prend, de jour en jour, plus d'importance. Les ouvrages se multiplient, sur Satan, le mythe d'Orphée, le thème de Prométhée, au point qu'il a semblé possible d'esquisser une histoire des mythes dans notre littérature. (Albouy 1969: 5)

Les références aux divinités de la fable, l'usage que les auteurs font des mythes hérités, l'étude des structures mythiques, tous ces travaux aideraient à mieux définir la nature et la fonction du mythe dans la littérature, ils font ressortir le cœur de l'acte d'écrire: signifiant polyvalent et plastique, disant ce qu'on dit et autre chose, se situant toujours sur plusieurs niveaux en même temps. Emprunté ou inventé, le mythe renferme le système des signes socioculturels et toutes les puissances du langage, il réanime, chez les grands écrivains, les archétypes les plus profonds, et par là, permet d'approcher encore du mystère de la création. La mythologie chinoise occupe dans la littérature une place importante, les textes anciens nous offrent un grand nombre de mythes et de légendes, les époques plus récentes nous apportent de nouveaux mythes. Yuan Ke (1985: 4) a classé les mythes, dont le nombre est de 3000 environ, en quatre catégories:

1. Êtres humains: dieux, héros mythiques, personnages légendaires ou historiques, immortels, démons et diables, familles nationales, etc.

2. Choses: celles qui révèlent un caractère mythique, par exemple, animaux, végétaux, minerais, médicaments, armes, instruments de musique.

3. Ciel et terre: espaces, étoiles, voie lactée (rivière céleste en chinois), vents et nuages au ciel; montagnes, rivières, villes, temples sur la terre.

4. Événements: le mythe raconte un événement, par exemple, Huit immortels passant la rivière, Porter la montagne pour rattraper le soleil, etc.

Ces mythes doivent aussi éveiller le plaisir des traducteurs dans leur recherche des techniques de recréation. Sans connaître la culture de la langue de départ et les lois intérieures des signes de mœurs, on ne peut réaliser une traduction satisfaisante. 
Dans la littérature chinoise, les citations-allusions sont nombreuses et constituent des moyens esthétiques très dynamiques et d'une expressivité riche. Les citationsallusions utilisent les personnalités historiques, les faits anciens et les propos des savants pour qualifier les faits présents. Dans la plupart des cas, elles forment des comparaisons qui dépassent la capacité d'expression lexicale. Par exemple, au lieu de dire «une personne très intelligente», on dit qu'elle est un «talent de sept pas» (七步之才), allusion faite à Cao Zhi, poète célèbre qui a réussi à composer un poème après avoir fait sept pas (durée très courte) ; quand on critique «un homme médiocre», on dit qu'il est «le A-dou» (阿斗), fils de Liu Bei sous les Trois Royaumes (iii ${ }^{e}$ siècle après J.-C.), qui était en fait un empereur complètement incapable. Ces citations sont courtes, de deux à cinq caractères en chinois, mais elles constituent des images concrètes et très métaphoriques. Elles sont tirées des textes anciens, se comptent par milliers et sont largement utilisées dans les textes actuels chinois. Dans la traduction du chinois en d'autres langues, ces citations créent de grandes difficultés.

\section{Signes des mœurs}

Les mœurs, dans toute civilisation, constituent une partie intégrante du patrimoine culturel. Elles ont contribué à maintenir les relations entre les individus et l'ordre social. Elles continuent à instruire les membres d'une communauté et à régulariser les comportements. Ce sont des systèmes de signes rigoureusement structurés et ordonnant la vie sociale. Par leur importance et leur diversité, les mœurs sont présentes dans tous les aspects sociaux et sont donc «des règles du comportement, des savoir-vivre et des savoir-faire [...] Elles sont les signes qui s'adaptent à toutes les circonstances de la communication: le temps, le lieu, le mode, l'efficacité, etc. » (Zhang 1988: 70-72). Les signes des mœurs dans les œuvres littéraires peuvent être envisagés de deux manières: premièrement, au sens strict du terme, il s'agit des règles de comportement que l'écrivain décrit dans son œuvre; deuxièmement, au sens large du terme, les mœurs désignent les œuvres littéraires elles-mêmes. Les six scènes dans la Comédie humaine sont conçues justement comme «études des mœurs». Dans le premier cas, nous pensons à des modes de vie: le vêtement, la nourriture, I'habitat, les moyens de déplacement, la symbolique des animaux, le «langage des fleurs», la kinésique et la proxémique, etc. Dans le second cas, nous pensons à des comportements sociaux et phénomènes sociaux décrits en signes esthétiques. Ces signes posent des problèmes spéciaux et demandent un traitement approprié dans la traduction.

\section{PROPOSITIONS DE TRAITEMENT}

Nous venons d'évoquer les signes sociaux, leurs rapports avec la littérature et une typologie simple des signes sociaux dans le texte littéraire. Ces réflexions ne se pré tendent pas exhaustives, elles servent juste à ouvrir les champs de réflexion. La pratique dans l'activité traduisante s'avère beaucoup plus importante. En partant des réflexions précédentes, nous essayons de proposer quelques traitements selon les types de signes à traduire. Depuis une vingtaine d'années en Chine, la traduction a connu une grand essor, des milliers d'ouvrages étrangers ont été traduits, notamment des œuvres littéraires. Avec le développement des activités traduisantes, naissent une série de théories de la traduction de type pragmatique ou scientifique. Récemment, 
I'introduction des théories étrangères nous fait découvrir des noms comme Nida, Mounin, Meschonnic, Catford, Pergnier, Steiner, Ladmiral, Seleskovitch, etc. Les théoriciens chinois s'affirment par leurs réflexions novatrices: «fidélité, expressivité et élégance» de Yan $\mathrm{Fu}$, «beautés sémantique, phonétique et formelle» de $\mathrm{Xu}$ Yuanchong, «niveau de la pensée, niveau de la langue et niveau de l'esthétique» de $\mathrm{Xu}$ Jun, etc. Ces recherches théoriques ont contribué à bâtir une théorie plus scientifique de la traduction et commencent à guider les traducteurs chinois dans leurs activités traduisantes.

\section{Rapporter le sens social connoté}

Dans le cadre de la traduction des signes sociaux, nous pourrons bénéficier des acquis des chercheurs cités ci-dessus. Pour les variantes des signes sociolinguistiques, nous devons les traduire en tenant compte du sens donné par le cadre social. Dans Peinture fraîche de Robert Escarpit, le bricoleur espagnol «crachant son mégot sur mes pieds, il toucha le bord de son vénérable béret: "Zé souis Pablo" », possède déjà une variante phonologique par son accent: il est étranger. Dans la traduction en chinois, il faut trouver l'expression qui cumule deux informations: Je m'appelle Paul et je suis étranger. Bien sûr, dans un tel cas, le traducteur peut recourir aux notes, cependant, l'excès de notes déplait au lecteur qui préfère une œuvre lisible à un texte plein de notes à déchiffrer. La variante grammaticale doit aussi être rendue dans la langue d'arrivée. Dans La Guerre des boutons de Louis Pergaud, nous lisons le dialogue suivant:

- Grouille toi, alors, je n'ai pas le temps de cotainer, moi!

- Y a du neuf?

- Ça se pourrait!

- Quoi?

- Viens toujours!

L'omission du sujet, la proposition d'un seul mot indiquent le milieu social du locuteur et son niveau culturel. Au fait, c'est un dialogue entre deux écoliers qui vont à l'école le jour de la rentrée. Dans la traduction en chinois, il faut y insérer la manière de parler des écoliers et leur registre de langue. Au niveau lexical, la variante doit aussi être prise en considération, le traducteur ne doit pas se contenter d'un dictionnaire bilingue, «il devra s'insinuer dans l'histoire, la tradition, la culture et même la vie sociale où a pris naissance l'œuvre littéraire qu'on envisage de traduire» (Xu 1997). Dans Le Deuxième Sexe de Simone de Beauvoir, nous lisons la phrase suivante: «La nature, l'amitié, quelques rencontres heureuses, un peu d'herbe le soir, une belle vie, libre et gaie.» L'herbe ici désigne le chanvre que les hippies des années soixante fumaient pour avoir des hallucinations enivrantes. Dans ce cas, il vaut mieux traduire «un peu d'herbe» par «fumer un peu de chanvre» en chinois. La traduction n'est pas une simple mise en place des signifiants dont les concepts sont déjà connus, elle suppose qu'on se familiarise avec un nouveau système sémiologique et sémantique et qu'on choisisse le signifiant approprié, parce que les termes qui désignent un objet ou une action peuvent avoir des connotations sociales. Quand nous traduisons un auteur étranger, nous ne traduisons pas simplement un lexique et une grammaire pleine de finesse, mais aussi une autre manière de percevoir le monde, une autre manière de représenter la vie. C'est cette façon différente d'appréhender la réalité, ce regard artistique qui sont difficiles à cerner et à traduire. 


\section{Transmettre la saveur originale}

Wang Yuelun propose dans "De la "coupe du nez et de l'œil" à la conservation de l'exotisme» (从 “削鼻剜眼” 到 “异国情调”) de préserver le mieux possible l'exotisme dans la traduction. On doit conserver non seulement le contenu, mais aussi les us et les coutumes, les éléments culturels et historiques étrangers, les archétypes, les figures, les expressions imagées, les citations littéraires, etc. (1984: 933-941). Dans la traduction des «idiomes», s'il existe des équivalents ayant la même image dans deux langues, la méthode générale est de prendre l'équivalent correspondant: les locutions «rusé comme un renard» et «blanc comme neige» peuvent être traitées de cette façon. S'il existe des équivalents avec une image différente dans les deux langues, on peut procéder de deux manières: soit on prend l'équivalent tout fait malgré la différence d'image, par exemple, on traduit «brebis galeuse» par «cheval qui nuit à son groupe» (害群之马), soit on gardel'image du texte de départ, par exemple, on traduit 《armé jusqu'aux dents» par 《武装到牙齿》 (armé jusqu'aux dents) au lieu de traduire par «armé comme tout» (全副武装), locution qui existe en chinois. Dans ce cas, à condition que cette traduction n'entraîne pas de confusion ou de malentendu, il faut garder l'image originale, car ceci nous permet de percevoir l'exotisme et d'enrichir le registre de locutions de la langue d'arrivée. Encore faut-il dire que l'expression «armé jusqu'aux dents» est déjà entrée dans la langue chinoise. Par contre, pour les locutions qui n'ont pas d'équivalents dans la langue d'arrivée, il faut les traduire en gardant l'image originale et en veillant à ce qu'elles soient compréhensibles dans la langue d'arrivée. Dans Thérèse Desqueyroux de M auriac, nous lisons le dialogue qui suit:

- Je recevrai demain l'avis officiel du non-lieu.

- II ne peut plus y avoir de surprise?

- Non : les carottes sont cuites, comme on dit.

«Les carottes sont cuites» n'a pas d'équivalent en chinois. Nous proposons de traduire cette locution par son image originale, car le contexte délimite le champ sémantique, il n'y aura pas de confusion. Si l'on traduit par une expression chinoise assez proche de cette expression : 《木已成舟》 (le bois est déjà devenu un bateau), on risque de brouiller le champ sémantique dont le découpage est différent pour ces deux expressions. Dans Bel ami de M aupassant, Forestier dit que «tous les hommes sont bêtes comme les oies et ignorants comme des carpes», nous préférons conserver les deux images originales dans la traduction en chinois. Car les deux comparaisons sont faciles à comprendre et feront connaître des expressions exotiques.

En ce qui concerne les mythes et citations-allusions, nous proposons de les traduire par un traitement à deux niveaux: d'abord, on procède à une traduction lexicale et phonologique selon les règles qu'on vient de citer; puis, si c'est nécessaire, on ajoute une note pour donner l'explication. Par exemple, on traduit Vénus par «Weinasi » (维纳斯) en chinois; de même, on traduit «嫦娥》 (déesse de la lune) par «Chang'e» en français avec une note aidant la compréhension. Dans les textes français, on rencontre souvent des expressions telles que «amour platonique», «vie épicurienne», «style balzacien», "esprit cartésien », ou les phrases telles que «La cigale n'a pas encore changé son habitude» (en parlant de la paresse de certains Français), «La fourmi reste toujours économe» (en désignant le manque de solidarité chez 
certains Français), etc. Ces expressions ou phrases venues des personnalités historiques ou de la culture française (Fables de La Fontaine) sont censées être connues de tous les Français cultivés; cependant, elles restent inconnues pour les lecteurs étrangers. Dans la traduction, il faut les traduire d'abord au niveau lexical, puis y ajouter une note qui fournit le fonds culturel: Platon, Épicure, Balzac, D escartes, «La cigale et la fourmi » de La Fontaine.

Les noms propres méritent aussi une réflexion pour trouver un bon traitement. Les noms propres, qu'ils soient de personnes ou de lieux, suivent le caractère arbitraire du signe linguistique, I'usage social les conventionnalise. M a Zhencheng (1996: 31-32), tout en signalant ce caractère arbitrai re, nous propose deux façons de traduire les noms propres. Pour les noms propres qui apparaissent peu fréquemment, on les traduit selon un tableau de transcription phonétique; pour les personnalités, s'il existe déjà une traduction, on suit la convention. Pour les noms de lieu, c'est le même traitement, sauf lorsque le nom a un sens dans la langue de départ et que ce sens participe à la formation de la signification globale du texte, par exemple, les noms de ville tels que «Phénix», «Oxford», «Trois-Rivières», «Belleville», etc. Pourtant un autre traitement est aussi admis, «Côte d'Ivoire» n'est plus traduit par «象牙海岸》 (côte où il y a des ivoires), mais par «Ketediwa» (科特迪瓦); 《Bordeaux» n'a jamais été traduit en 《水边 » (au bord des eaux). Toutefois, pour certains noms propres dont le sens participe à la sémantique de l'œuvre, nous préférons les traduire sémantiquement. Par exemple, dans la nouvelle version en chinois de Notre-Dame de Paris, 《Fleur de Lys», «Colombe», «Chantefleur 》 sont traduits en 《百合花》, 《小白鸽》, 《香的花》 (fleur qui sent bon). Nous estimons que la traduction sémantique peut mieux garder la saveur originale. Les noms de personnes en chinois, tout comme les noms de famille français, forment un système complexe et riche en signification. Nous essayons de les représenter en langue d'arrivée en y mettant des marques culturelles. Les noms chinois sont traduits actuellement selon un ordre: nom de famille d'abord et prénom ensuite, ce qui est différent des Occidentaux. Et puis la généralisation du 《拼音»(Pingying: transcription alphabétique) impose des marques typographiques propres au chinois; les noms comme "Zhang Jianguo», "Yang Dongfang» ou «Li Dapeng» sensibilisent les lecteurs occidentaux à la phonétique et à la morphologie. À force de lire, le lecteur arrivera à reconnaître les noms chinois et se situera dans la culture chinoise.

\section{Interpréter les signes des mœurs}

Les signes des mœurs dans les œuvres littéraires sont représentés par des signes linguistiques, ils sont à interpréter. Le procédé comporte deux phases: une phase de traduction linguistique, une phase de notation interprétative. Pendant la phase de traduction linguistique, on traduit les signes de mœurs au niveau lexical, sans mettre trop d'éléments qui troublent la lisibilité du texte, ensuite on passe à la deuxième phase. La notation interprétative peut se faire soit dans le texte, soit sous forme de notes extratextuelles. Dans le premier cas, il s'agit plutôt d'un ajout intertextuel. Par exemple, l'acte de «hocher la tête» peut causer la confusion en chinois: «remuer la tête de haut en bas» veut dire «d'accord » en chinois, tandis que «remuer la tête de gauche à droite» veut dire «non », le sens de ce geste est tout à fait contraire chez les Grecs. Là, nous préférons traduire par «hocher la tête en signe de dénégation». 
Apporter des chrysanthèmes à des funérailles est un fait courant en Occident, il vaudrait mieux ajouter «en signe de recueillement», parce qu'en Chine, le chrysanthème est le signe de l'élégance. Le retard volontaire d'un invité à un dîner doit être interprété comme «signe de politesse» vis-à-vis de la maîtresse de la maison, car en Chine, le retard au rendez-vous chez un ami est justement un acte impoli. Dans le deuxième cas, on est obligé de recourir aux notes en bas de page malgré certains inconvénients de la méthode, parce que sans interprétation, la compréhension risque d'être faussée et la traduction échoue. De plus, les mœurs sont des systèmes de signes et chaque système possède sa cohérence et son harmonie d'ensemble. Ces systèmes doivent être traités différemment dans la traduction; la réussite dépend du savoir, des compétences et très souvent, du génie créateur du traducteur.

\section{NOTES}

1. Lucien Goldmann, Le Dieu caché, 1955, p. 16. La présente citation est une reconversion du chinois en français; pour être précis, nous renverrons le lecteur à l'œuvre originale.

2. Gustave Flaubert, Madame Bovary, Paris, Le Livre de poche, 1972, p. 167-168.

\section{RÉFÉRENCES}

Al bouy, P. (1969) : Mythes et mythologies dans la littérature française, Paris, Armand Colin. Bart hes, Roland (1977) : Poétique du récit, Paris, Seuil, «Points».

Gr eimas, A. J. (1973) : Sémiotique narrative et textuelle, Paris, Larousse.

Guir aud, Pierre (1983) : La Sémiologie, Paris, Presses Universitaires de France.

H ymes, D. (1974) : «Anthropology and Sociology», Current Trends in Linguistics, vol. 12, La Haye, Mouton.

M a, Zhencheng (1996) : «La traduction phonétique est une convention», dans Xu Jun, Écriture, littérature et culture, Presses de I'Université de Nanjing.

M ounin, Georges (1970) : Introduction à la sémiologie, Paris, Éditions de Minuit.

Q u en eau, Raymond (1982) : Exercices de style, Paris, Gallimard.

Tyl er (1969) : Cognitive Anthropology, N ew York, Montréal, Holt, Rinehart and Winston.

Wang, Yuelun (1984) : «De la "coupe du nez et de l'œil" à la conservation de l'exotisme», Essais de traduction, rédigé par Luo Xinzhang, Presse commerciale.

Xu, Jun (1992): «Connotation et traduction», Critique de la traduction littéraire, Nanjing, Éditions Yilin.

Xu, Zhenhua (1997) : Traduction: différence linguistique et différence cognitive, Colloque international de Shanghai sur la traduction, juin.

Yuan, Ke (1985) : Dictionnaire des mythes et légendes chinois, Édition des dictionnaires de Shanghai.

Zhang, Xinmu (1988) : La Sémiologie et les M œurs, Thèse de maîtrise, Université de Nankin.

- - (1989) : «Sémiologie de la nourriture», Beijing Information, n 34, p. 24.

- - (1997) : «Approche sémiologique de la traduction », Traduire, SFT, nº 172, p. 19. 